\title{
A Unique Case of Replantation of Previously Replanted Fingers
}

\author{
Ravindra Bharathi ${ }^{1}$ Praveen Bhardwaj ${ }^{1}$ Vigneswaran Varadharajan ${ }^{1}$ Hari Venkatramani ${ }^{1}$ \\ S. Raja Sabapathy ${ }^{1}$ \\ ${ }^{1}$ Department of Plastic, Hand, Burns and Reconstructive \\ Microsurgery, Ganga Hospital, Coimbatore, Tamil Nadu, India \\ Address for correspondence Ravindra Bharathi, MS, MCh, \\ Ganga Hospital, Coimbatore, Tamil Nadu, India \\ (e-mail: prabharathi@hotmail.com, rajahand@gmail.com).
}

Indian J Plast Surg:2021;54:86-89.

\begin{abstract}
Replantation of digital amputations is now the accepted standard of care. However, rarely will a replantation surgeon be presented with amputated fingers which have been previously replanted. In our literature search, we could find only one publication where a replanted thumb suffered amputation and was successfully replanted again. We report the technical challenges and the outcome of replanting two fingers which

Keywords

- second time replantation

- multiple finger amputation

- re-replantation

- digital amputation

- finger replant suffered amputation 40 months after the initial replantation and were successfully replanted again. Replantation was critical since the amputated fingers were the only two complete fingers in that hand which had initially suffered a four-finger amputation. The second-time replantation of previously replanted fingers is reported to allay the concern of the reconstructive surgeon when faced with this unique situation of "repeat amputation of the replanted finger." Second-time replantation is feasible and is associated with high-patient satisfaction. Replantation must be attempted especially in the event of multiple digit amputations.
\end{abstract}

\section{Introduction}

Replantation of digital amputations is now the accepted standard of care. When they involve multiple digits, it becomes an absolute indication..$^{1-3}$ Rarely will a replantation surgeon be presented with amputated fingers which have been previously replanted. We could find only one report of such unusual situation in the literature where a replanted thumb suffered amputation and was successfully replanted again. ${ }^{4}$ We are herein reporting a rare case of replantation of two fingers which suffered amputation 40 months after the initial replantation and were successfully replanted again. Replantation was critical since the amputated fingers were the only two complete fingers in that hand which had initially suffered a four-finger amputation. The technical challenges and recommendations to tackle this unusual situation are discussed. The patient was highly satisfied with the outcome.

published online

March 4, 2021
DoI https://doi.org/

$10.1055 / \mathrm{s}-0041-1723911$ ISSN 0970-0358.

\section{Case Report}

On November 11, 2014, a 34-year-old male, right hand dominant worker sustained amputation of all four fingers of his left hand in a paper cutting machine and presented to us 4 hours after the injury. The level of amputation of the index, middle, ring and little fingers were through the shaft of proximal phalanx, neck of proximal phalanx, shaft of middle phalanx, and at the distal interphalangeal joint, respectively (-Fig. 1). The amputated parts were brought well-preserved. The option of replantation was presented to the patient and following discussion, replantation of the index and middle fingers and closure of the amputation stump of the lesser injured ring and little fingers were decided. The patient underwent replantation of the index and middle fingers; the bone was shortened at the proximal phalanx level while preserving the metacarpophalangeal (MCP) and proximal interphalangeal (PIP) joints; stabilization was performed using

\footnotetext{
(C) 2021. Association of Plastic Surgeons of India.

This is an open access article published by Thieme under the terms of the Creative Commons Attribution-NonDerivative-NonCommercial-License, permitting copying and reproduction so long as the original work is given appropriate credit. Contents may not be used for commercial purposes, or adapted, remixed, transformed or built upon. (https://creativecommons.org/licenses/by-nc-nd/4.0/).

Thieme Medical and Scientific Publishers Pvt. Ltd. A-12, 2nd Floor, Sector 2, Noida-201301 UP, India
} 
axial Kirschner wire, followed by the repair of the flexor digitorum profundus (FDP) tendon, the extensor tendon, two dorsal veins and both digital arteries and nerves of each digit. Flexor digitorum superficialis (FDS) was not repaired. The ring and little fingers underwent closure of the amputation stump, preserving the PIP joint. Both the replanted digits survived. The Kirschner wires were removed at 6 weeks and the patient was started on supervised physiotherapy and regular follow-up visits ( - Fig. 2).

Five months later, he returned to his previous occupation with the paper cutting machine. When reviewed at 11 months postreplantation, he had developed protective sensation in both the index and middle fingers and was using the hand well for day-to-day activities and at work.

On March 12, 2018, he injured his left hand again while working with the same paper cutting machine and presented to us 3 hours after the injury. Examination revealed guillotine amputation of the replanted index finger through the MCP joint and the middle finger through the previous replantation level. The amputation was obliquely oriented with the amputated fingers connected through skin flap of second web space ( - Fig. 3 ). After exploration of the amputated parts and the stumps, replantation of the index and middle fingers was performed with arthrodesis of the MCP joint of the index finger and fixation of the proximal phalanx of the middle finger (after $5 \mathrm{~mm}$ shortening) with axial Kirschner wires, repair of extensor tendons, one dorsal vein, radial digital artery and nerve of each digit. We could not repair the ulnar digital nerve, as its distal cut end could not be identified. The vein and artery repair sites were just proximal to the previous replantation site and could be performed in usual manner with immediate good distal flow and survival of the digits. Contrary to our expectation and concern about the fibrosis surrounding the vessels at the previous replant site, the dissection of the vessels and the tissues was as normal as in the first-time replant. However, the pull on the distal end of the flexor tendon revealed adhesions distally; hence, we could not perform the flexor tendon repair and decided for secondary flexor tendon reconstruction. At 6 weeks, the Kirschner wires were removed. Fusion of the MCP joint of index finger was found to be complete in the radiographs taken at 3 months following replantation. The patient was trained for thumb opposition to the middle finger. At 4 months, he was offered secondary flexor tendon reconstruction of the index and middle fingers. He did not return for the procedure.

At 19 months after the second replantation, the patient was called back for review and assessment recommended for evaluation of outcomes following digital replantation. ${ }^{5}$ Subjective outcome was assessed with Michigan hand outcome questionnaire (MHQ). Two-point discrimination, static and dynamic, was $6 \mathrm{~mm}$ and $4 \mathrm{~mm}$ respectively for the middle finger. He could only perceive one point beyond $15 \mathrm{~mm}$ with the index finger. There was no active motion present in the index finger, and the middle finger had $20^{\circ}$ motion at the MCP joint only. The grip strength

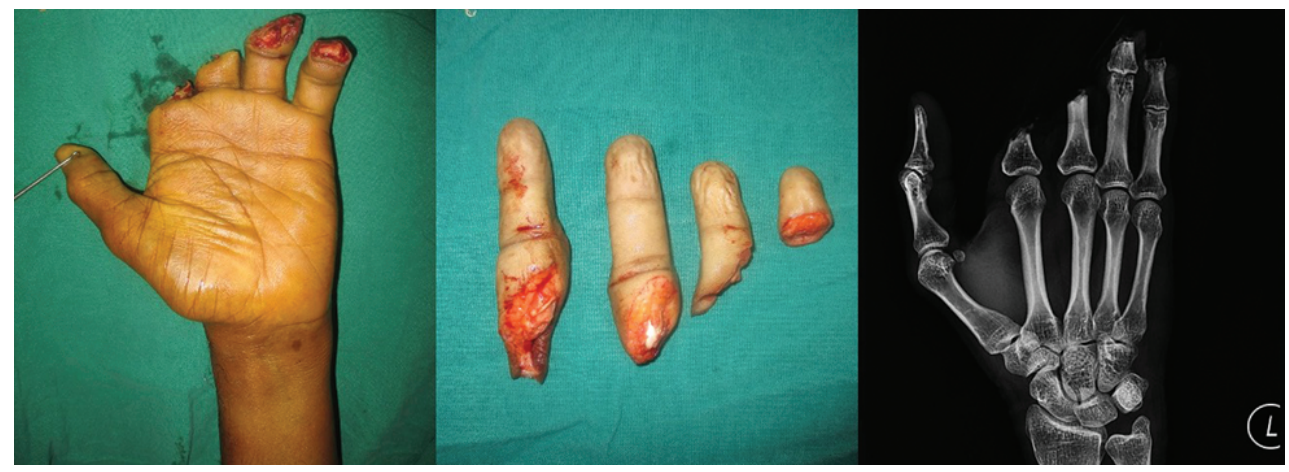

Fig. 1 Guillotine amputation of the index, middle, ring and little fingers of left hand through the shaft of proximal phalanx, neck of proximal phalanx, shaft of middle phalanx and distal interphalangeal (DIP) joint, respectively.

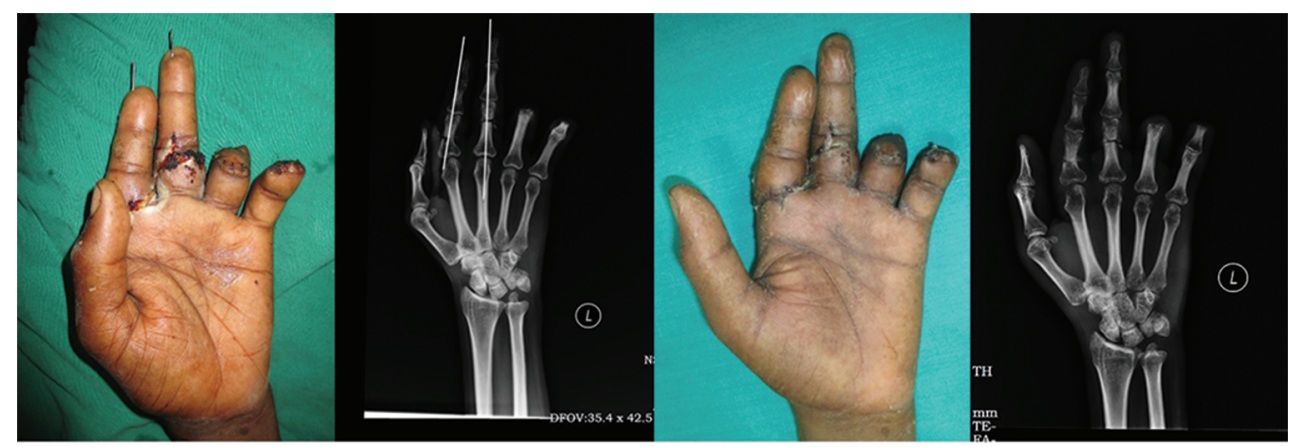

Fig. 2 Clinical photo and the corresponding radiograph at 2 weeks and 6 weeks postreplantation. 
of the left hand measured $9.3 \mathrm{kgs}$, which was $25.8 \%$ of the normal hand. He was able to generate pulp to pulp pinch of $1.5 \mathrm{kgs}$ ( $5.5 \mathrm{kgs}$ in right hand), pulp to lateral pinch of $4.5 \mathrm{kgs}$ ( $7.5 \mathrm{kgs}$ in right hand), and tripod pinch of $2.5 \mathrm{kgs}$ ( $6.5 \mathrm{kgs}$ in right hand). No nail atrophy, pain, or cold intolerance was noted. Subjective assessment with MHQ revealed scores of 86 compared with 96 in his right hand. He was able to oppose the thumb to the middle fingertip ( - Fig. 4).

However, he did not return to his previous job with the paper cutting machine and instead worked as a supervisor in the same workplace. He was pleased with the results of the second replant and was not keen for the secondary flexor reconstruction, as he was managing his daily activities at home and work with ease.

\section{Discussion}

Replantation of the amputated digits is a standard operation with 70 to $90 \%$ survival rate. ${ }^{6}$ However, replantation of the replanted digits-"second-time replantation" is a very unique situation. We could only retrieve a single case report of this rare situation in hand surgery literature. Tsai, in 1979, reported a second-time replantation of previously replanted thumb of a patient who had amputation of all the digits of the left hand and right thumb during the first injury, which were replanted. At 21 months postreplantation, he had amputation of the previously replanted left thumb, which was again successfully replanted. ${ }^{4}$ Our report is probably the first, where two fingers have undergone "second-time replantation."

Two cases of successful second time replantation of penile amputation have been reported. ${ }^{7,8}$ Both these cases were attributed to the psychiatric illness of the patients where the patients had voluntarily stopped the medications. Some question whether it is worth the while to do the second-time replant in psychiatric patients with self-inflicted injury. Even in them, it is thought to be worth under the cover of proper medication. ${ }^{8}$ In actively working people, it is a definite indication.

Concerns could arise in the mind of the surgeon about the technical difficulties that could arise when the amputation

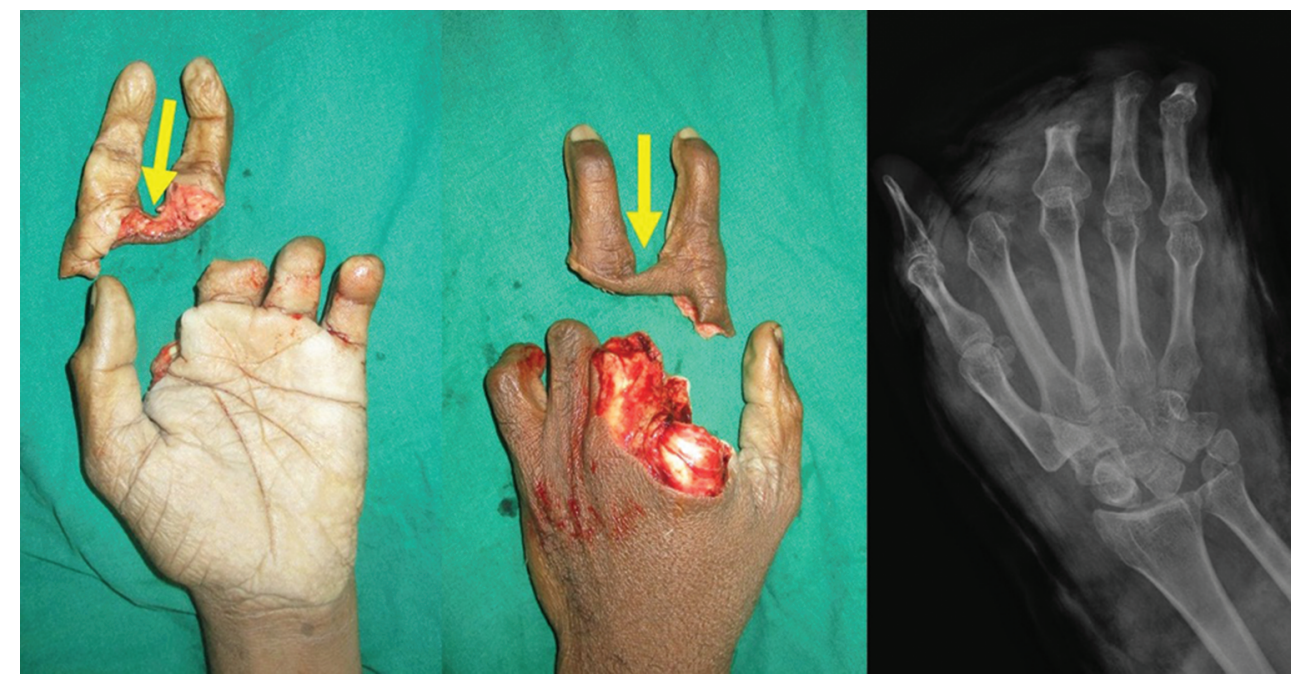

Fig. 3 Second time amputation of the replanted index and middle fingers. Note the intact web between the amputated fingers (arrow).

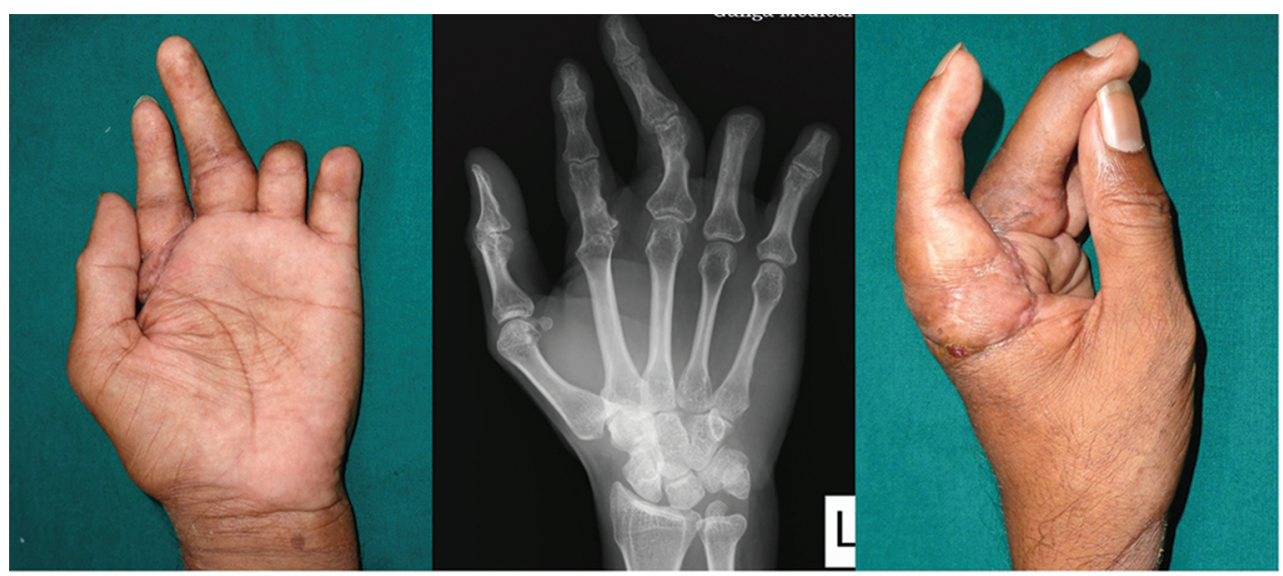

Fig. 4 Clinical photos and radiograph at 19 months following second replantation. Radiograph shows good fusion at the metacarpophalangeal (MCP) joint of index and bone union at the proximal phalanx of the middle finger. Patient was using the hand well with the middle finger and thumb opposition pinch. 
level is close to the previous replant site. Shortening of the bone could be preferentially done on the side which allows one to go away from the previous replant site, in order to avoid probable fibrosis in that area. In our case, the reamputation was just a centimeter proximal to the previous anastomosis site. Proximal shortening by arthrodesis of the MCP joint of the index finger and proximal phalanx of the middle finger allowed easy reach of the vessels for end-to-end repair without need of a vein graft. Preferential shortening of the bone on the side away from the previous anastomosis site is an important technical consideration to get an easy end-to-end repair without disturbing the previous anastomosis sites. Vessel dissection was easy and there was no fibrosis to cause difficulty in dissection of the vessels. On the contrary, the adhesions in the flexor tendon precluded repair of the flexor tendons. Although it is technically possible to perform flexor tenolysis distally and do a flexor tendon repair, we avoided it, because the present amputation was about a cm proximal to the previous replantation site, and we wanted to avoid extended dissection into the previous vessel repair sites with deranged anatomy. Furthermore, a tenolysed flexor tendon need early movement, and in a replanted finger, we would not have been able to do that as would be needed. Hence, we avoided it and left it for later reconstruction. This case also showed that good sensory recovery can be achieved by second-time repair of the nerve. Repair of only one digital nerve (radial) provided good sensory recovery at the fingertip in this case, probably because of the cross-innervation facilitated by collateral sprouting and unmasking of ineffective synaptic connections at the fingertip observed by others. ${ }^{9}$ The middle finger showed excellent recovery of sensation, but we were not able to decipher the cause of the poor recovery in the index finger. The good mobile thumb acting against the stable index and middle fingers helped him to function. The unintentional radial deviation deformity at the middle finger in this case was rather useful, as it allowed better opposition of this less mobile digit to the thumb and facilitated pinch and grasp function.

Retrospective analysis of the original injury showed that we should have persisted with the replantation of the ring and the little fingers, since they were technically possible. ${ }^{10}$ With the amputation of the index and the middle in the second accident, it became more critical that we achieve success with the second replant in these fingers, since the ring and little were short.

The patient has returned to his previous occupation at 5 months of replantation in the first instance and to a different job 3 months following the second replantation. The high MHQ scores achieved by the patient indicates the mere presence of the fingers helped him to return to the society in a faster and smoother manner. The recently concluded FRANCHISE study, a multicenter international retrospective cohort study, also confirms that whenever adequate infrastructure is available, with proper patient selection, replantation is recommended and is cost-effective when three or more fingers are amputated. ${ }^{11}$

\section{Conclusion}

The second-time replantation of previously replanted fingers is feasible. This case is reported to allay the concern of the reconstructive surgeon when faced with this unique situation of "repeat amputation of the replanted finger." This patient reported high satisfaction with the procedure. Replantation must be attempted, especially in the event of multiple digit amputations.

\section{Conflicts of Interest}

None declared.

\section{References}

1 Chung K, Alderman A. Replantation of the upper extremity: indications and outcomes. J Am Soc Surg Hand 2002;2:78-94

2 Sabapathy SR, Venkatramani H, Bharathi RR, Bhardwaj P. Replantation surgery. J Hand Surg Am 2011;36(6):1104-1110

3 Chim H, Maricevich MA, Carlsen BT, et al. Challenges in replantation of complex amputations. Semin Plast Surg 2013; 27(4):182-189

4 Tsai T. A complex reimplantation of digits: a case report. J Hand Surg Am 1979;4(2):145-149

5 Sebastin SJ, Chung KC. Challenges in measuring outcomes following digital replantation. Semin Plast Surg 2013;27(4): 174-181

6 Dec W. A meta-analysis of success rates for digit replantation. Tech Hand Up Extrem Surg 2006;10(3):124-129

7 Fujiki M, Ozaki M, Kai A, Takushima A, Harii K. Successful second microsurgical replantation for amputated penis. Plast Reconstr Surg Glob Open 2017;5(9):e1512

8 Volkmer BG, Maier S. Successful penile replantation following autoamputation: twice! Int J Impot Res 2002;14(3):197-198

9 Jain A, Dunlop R, Hems T, Tang JB. Outcomes of surgical repair of a single digital nerve in adults. J Hand Surg Eur Vol 2019; 44(6):560-565

10 Venkatramani H, Sabapathy SR. Fingertip replantation: technical considerations and outcome analysis of 24 consecutive fingertip replantations. Indian J Plast Surg 2011;44(2):237-245

11 Chung KC, Yoon AP, Malay S, Shauver MJ, Wang L, Kaur S; FRANCHISE Group. Patient-reported and functional outcomes after revision amputation and replantation of digit amputations: The FRANCHISE Multicenter International Retrospective Cohort Study. JAMA Surg 2019;154(7):637-646 